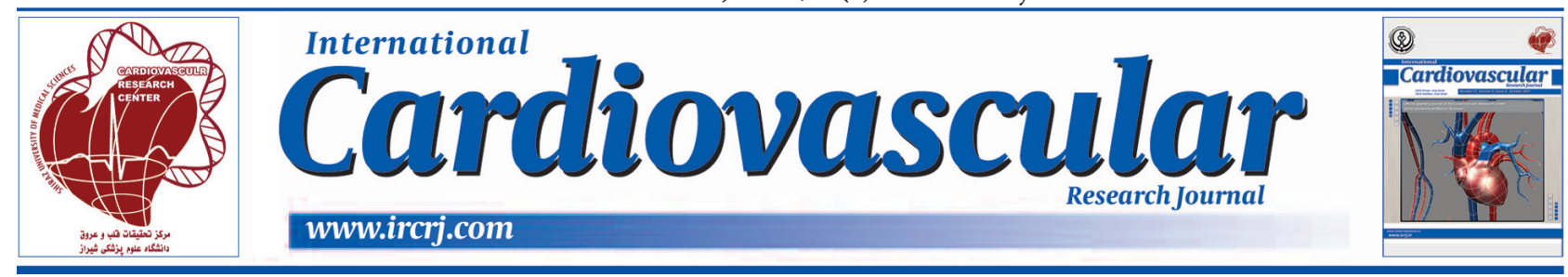

\title{
The Predictors of No-Reflow Phenomenon after Primary Angioplasty for Acute Myocardial Infarction
}

\author{
Kamran Aeinfar ${ }^{1}$, Ata Firouzi ${ }^{2, *}$, Hossein Shahsavari ${ }^{1}$, Hamidreza Sanati ${ }^{2}$, Reza Kiani ${ }^{2}$, Farshad \\ Shakerian ${ }^{2}$, Ali Zahed Mehr ${ }^{2}$
}

${ }^{1}$ Rajaie Cardiovascular Medical and Research Center, Iran University of Medical Sciences, Tehran, IR Iran

${ }^{2}$ Cardiovascular Intervention Research Center, Rajaie Cardiovascular Medical and Research Center, Iran University of Medical Sciences, Tehran, IR Iran

\begin{tabular}{l}
\hline A R T I C L E I N F O \\
\hline Article Type: \\
Review Article \\
\hline
\end{tabular}

Article History:

Received: 11 Jul 2015

Revised: 05 Jan 2016

Accepted: 11 Jan 2016

\section{Keywords:}

Myocardial Infarction

Angioplasty

Balloon

No-Reflow Phenomenon

Myocardial Reperfusion

\begin{abstract}
A B S TR A C T
Background: No-reflow phenomenon is a serious complication of primary Percutaneous Coronary Intervention (PCI), which may increase the risk of progressive myocardial damage, profound left ventricular dysfunction, and death.

Objectives: This study aimed to investigate the incidence of no-reflow phenomenon and its clinical, para-clinical, and angiographic determinants in patients who underwent primary PCI for ST Elevation Myocardial Infarction (STEMI).

Patients and Methods: This non-randomized prospective cohort study was conducted on 397 patients in a cardiovascular tertiary care center in Tehran, Iran from April 2012 to April 2014. The inclusion criteria of the study were presenting with acute STEMI of $\leq 12$ $\mathrm{h}$ duration or having admitted between 12 and 24 hours after onset with symptoms and signs of ongoing ischemia. The participants underwent standard coronary angiography. No-reflow phenomenon was defined as a Thrombolysis In Myocardial Infarction (TIMI) flow $\leq 2$ and no presence of spasm, distal embolization, or dissection after completion of the procedure. The association between no-reflow and its determinants was assessed by chi-square, student's t-test, or Mann-Whitney U test. Logistic regression models were also used for multivariate analysis. $\mathrm{P}$ values $<0.05$ were considered to be statistically significant.

Results: The participants' mean (SD) age was of 59 (12.2) years and female/male ratio was $83 / 314$. The incidence of no-reflow phenomenon was 63 (15.9\%). Besides, the results of multivariate analysis showed that only thrombus burden, lesion length, time to reperfusion, and type of occlusion had an adjusted association with this phenomenon. Conclusions: The study results suggested that no-reflow phenomenon after primary PCI would be predictable. Thus, preventive measures, such as using distal protective devices or administration of platelet glycoprotein IIb/IIIa antagonists, are advised to be used in high-risk patients.
\end{abstract}

Implication for health policy/practice/research/medical education:

Considering the results of this study, cardiologists may be able to predict the occurrence and apply suitable methods for prevention of no-reflow phenomenon in patients with STEMI after primary PCI.

\section{Background}

Nowadays, primary Percutaneous Coronary Intervention (PCI) is a gold standard treatment for patients with acute ST Elevation Myocardial Infarction (STEMI) (1). Although

*Corresponding author: Ata Firouzi, Rajaie Cardiovascular Medical and Research Center, Vali-e-Asr St., Niayesh Blvd, Tehran, IR Iran. Tel: +98-2123922174, Fax: +98-2122663217,

E-mail: atafirouzi@yahoo.com the full patency of the infarct-related artery is achieved by primary PCI most of the times, the success rate can be reduced if the no-reflow phenomenon occurs $(2,3)$. Thrombolysis In Myocardial Infarction (TIMI) flow grade is the most commonly used method for assessment of primary PCI success (4). No-reflow phenomenon is a serious complication of primary PCI defined as a TIMI 
flow $\leq 2(2,3)$. This phenomenon can occur after primary PCI in up to one fourths of cases and such patients are at an increased risk of progressive myocardial damage, profound left ventricular dysfunction, and higher morbidity and mortality rates $(2,3,5-7)$. Up to now, many studies have investigated the pathogenesis and risk factors of no-reflow phenomenon (5-10).

\section{Objectives}

The present study aims to investigate the clinical, laboratory, and angiographic predictors of no-reflow phenomenon in patients who underwent primary PCI for acute STEMI in a tertiary referral cardiovascular center.

\section{Patients and Methods}

\subsection{Study Participants}

This non-randomized prospective cohort study was conducted in Rajaie Cardiovascular Medical and Research Center, the main cardiovascular tertiary care center in Tehran, Iran, from April 2012 to April 2014. Totally, 397 patients admitted in our center with a diagnosis of acute STEMI who underwent emergent coronary catheterization were consecutively enrolled into the study. According to the 2013 ACCF/AHA Guideline for the Management of ST-Elevation Myocardial Infarction, "STEMI is a clinical syndrome defined by characteristic symptoms of myocardial ischemia in association with persistent Electrocardiographic (ECG) ST elevation and subsequent release of biomarkers of myocardial necrosis" (1). The inclusion criteria of the study were presenting with acute STEMI of $\leq 12 \mathrm{~h}$ duration or having admitted between 12 and 24 hours after onset with symptoms and signs of ongoing ischemia. After coronary angiography, the patients with coronary vasospasm, less than 50\% diameter stenosis, and need for emergent/surgical revascularization, including left main coronary stenosis and any lesions leading to MI on the grafted vessels in the patients previously undergone $\mathrm{CABG}$, patients who received platelet glycoprotein IIb/IIIa receptor antagonists before angiography, and those who were treated with thrombolytic therapy, were excluded from the study. The study protocol was approved by the research and ethics committee of Rajaie Cardiovascular Medical and Research Center.

\subsection{Angiography and Primary PCI Procedure}

All the patients were treated with 300 milligram (mg) aspirin, $600 \mathrm{mg}$ clopidogrel, and 5000 - 10000 units unfractionated heparin before transmission to catheterization laboratory. Standard left and right coronary angiography was performed in all the patients via femoral approach using guiding catheter and at least 2 best projections were acquired for each patient. The angiograms were assessed and quantitative angiographic measurements were done by an expert interventional cardiologist and the following findings were recorded: Infarct Related Artery (IRA) and its luminal diameter, target lesion characteristics (lesion location, lesion length, type of occlusion, lesion type in subtotal occlusion, and thrombus burden), and initial and post procedural TIMI flow grade. Decision to perform primary PCI and to choose the optimal PCI technique in each patient was also made by the interventional cardiologist after coronary angiography.
Depending on the lesion characteristics, one of the following techniques was used for primary PCI: balloon angioplasty, stenting with pre-dilation, and direct stenting with or without thrombus suction.

Occlusion was defined as total type if the lesion had an abrupt end without tapering and as subtotal if the lesion had a tapered end. If the most protruding margin of the lesion was located in the outer quarter of the vessel lumen, the lesion was defined as subtotal. Besides, the intraluminal filling defects that did not have calcification were defined as thrombus (9). The thrombus burden of the target lesion was assessed according to the TIMI thrombus classification (4). "Thrombus burden was classified as mild if consistent with TIMI thrombus class 0 and 1 , moderate if consistent with TIMI thrombus class 2 and 3, and high if the greatest linear dimension of the thrombus was more than TIMI thrombus class 3" (9).

\subsection{Definition of No-Reflow Phenomenon}

If TIMI flow $\leq 2$ was observed despite successful dilatation and there was no spasm, distal embolization, or dissection after completion of the procedure, the patient was considered to have no-reflow phenomenon.

\subsection{Reperfusion Time}

Time to reperfusion was defined as the time from the onset of symptoms to the first angioplasty balloon inflation. Additionally, door to balloon was defined as the time from patient's arrival to the emergency department to the first balloon inflation (1).

\subsection{Study Variables and Data Collection}

The primary outcome of the study was "no-reflow phenomenon". Other variables, including the predictors of the no-reflow phenomenon, were measured, as well. Besides, demographic data, risk factors of cardiovascular diseases, clinical and para-clinical findings, and characteristics of the recent MI were extracted from the patients' medical records. Also, angiographic findings, primary PCI findings, and characteristics of the target lesion were obtained during the angiographic procedure, as explained above.

\subsection{Statistical Analysis}

All the statistical analyses were performed using the IBM SPSS statistical software for Windows, version 19.0 (IBM Corp., Armonk, NY, USA). Firstly, KolmogorovSmirnov test was used to assess the normal distribution of the data. Categorical variables were expressed as number and percentage and quantitative ones as mean (standard deviation) or median (interquartile range) for the variables that did not follow normal distribution. Categorical variables were compared by chi-square, student t-test, or Mann-Whitney test, as appropriated. In addition, binary logistic regression analysis was used for multivariate analysis. $\mathrm{P}$ values $<0.05$ were considered to be statistically significant.

\section{Results}

\subsection{Baseline Characteristics}

This study was conducted on 397 patients with the mean 
age of $59 \pm 12.2$ years (female/male ratio $=83 / 314)$. Noreflow phenomenon was diagnosed in 63 patients $(15.9 \%)$. The patients with and without the no-reflow phenomenon were compared regarding the background data, and the results have been presented in Table 1 . Accordingly, the patients in the no-reflow group were older than other participants $(\mathrm{P}<0.001)$. The history of MI was also higher among these patients $(\mathrm{P}=0.001)$.

\subsection{Characteristics of Myocardial Infarction}

Some characteristics of MI in our study population have been presented in Table 2. Based on the results, there was no significant difference between the two groups regarding the risk factors of coronary artery disease, history of previous MI, and the infarction territory in the current MI and IRA. However, time to reperfusion and door to balloon time were longer in the no-reflow group $(\mathrm{P}<0.001)$. Except for platelet count, inflammatory factors were also higher among the patients with the no-reflow phenomenon $(\mathrm{P}<$ 0.001) (Table 2).

\subsection{Coronary Angiography Findings}

The associations between the incidence of no-reflow phenomenon and its determinants evaluated by coronary angiography have been presented in Table 3. Except for the culprit artery, all the measured factors were significantly associated with the no-reflow phenomenon. In the no-reflow patients, the target lesion was mainly located in the proximal segment of the arteries and occlusion was mainly total and with greater lesion length. Thrombosuction was a frequent method for reperfusion in these patients. Prescription of GP $\mathrm{IIb} /$ IIIa inhibitor was also more frequent in the patients with no-reflow phenomenon (all P values $<0.001$ ).

\subsection{Multivariable Analysis}

For assessment of the adjusted associations between the no-reflow phenomenon and other predictors detected in bivariate analysis, a logistic regression model with backward elimination method was applied and the results have been presented in Table 4 . The results indicated that among the several predictors previously mentioned, only

\begin{tabular}{llll}
\hline Table 1. Comparison of the Two Groups Regarding the Baseline Data & \\
\hline & Normal Reflow, $(\mathbf{n}=\mathbf{3 3 4})$ & No-Reflow, $(\mathbf{n}=\mathbf{6 3})$ & P value \\
\hline Age, years & $64(13.8)$ & $58(11.6)$ & $<0.001$ \\
Gender (F/M) & $15 / 48$ & $68 / 266$ & 0.3 \\
Hypertension & $22(34.9 \%)$ & $115(34.3 \%)$ & 0.5 \\
Diabetes mellitus & $12(19 \%)$ & $65(19.5 \%)$ & 0.7 \\
Smoking & $7(11.1 \%)$ & $39(12 \%)$ & 0.3 \\
Hyperlipidemia & $21(33.3 \%)$ & $109(32.6 \%)$ & 0.1 \\
Family history & $3(4.7 \%)$ & $17(5 \%)$ & 0.2 \\
History of MI & $30(47.6 \%)$ & $55(16.5 \%)$ & 0.001 \\
\hline
\end{tabular}

Abbreviations: MI, myocardial infarction

${ }^{*}$ Data presented as mean (SD) and count (\%)

\begin{tabular}{|c|c|c|c|}
\hline & No-Reflow, $(n=63)$ & Normal Reflow, $(n=334)$ & P value \\
\hline \multicolumn{4}{|l|}{ MI territory } \\
\hline Anterior & $15(23.8 \%)$ & $62(18.6 \%)$ & \multirow{3}{*}{0.2} \\
\hline Inferior & $47(74.6 \%)$ & $253(75.7 \%)$ & \\
\hline Posterior & $1(1.6 \%)$ & $19(5.7 \%)$ & \\
\hline \multicolumn{4}{|c|}{ Time to reperfusion } \\
\hline$\leq 2$ hours & $0(0)$ & $89(26.6)$ & \multirow{3}{*}{$<0.001$} \\
\hline 2 - 4 hours & $2(3.2)$ & $157(47)$ & \\
\hline$\leq 4$ hours & $61(96.8)$ & $88(26.3)$ & \\
\hline \multicolumn{4}{|c|}{ Door to balloon } \\
\hline$<30 \mathrm{~min}$ & $1(1.6 \%)$ & $129(38.6 \%)$ & \multirow{3}{*}{$<0.001$} \\
\hline $30-60 \mathrm{~min}$ & $19(30.2 \%)$ & $161(48.2 \%)$ & \\
\hline$\geq 60 \mathrm{~min}$ & $43(68.3 \%)$ & $44(13.2 \%)$ & \\
\hline \multicolumn{4}{|c|}{ ST segment resolution } \\
\hline$\geq 70 \%$ & $22(34.9 \%)$ & $264(79 \%)$ & \multirow{2}{*}{$<0.001$} \\
\hline$<70 \%$ & $41(65.1 \%)$ & $70(21 \%)$ & \\
\hline CTnI & $33.7(21)$ & $1.8(3)$ & $<0.001$ \\
\hline CK-MB & $218(106)$ & $45(25.3)$ & $<0.001$ \\
\hline WBC count & $15800(400)$ & $10300(3023)$ & $<0.001$ \\
\hline Platelet count & $219000(3000)$ & $219000(66250)$ & 0.3 \\
\hline ESR & $63(36)$ & $17(9)$ & $<0.001$ \\
\hline hs CRP & $29(26)$ & $4(4)$ & $<0.001$ \\
\hline
\end{tabular}

Abbreviations: MI, myocardial infarction; CTnI, cardiac troponin I; CK-MB, creatine kinase-MB; WBC, white blood cell; ESR, erythrocyte sedimentation rate; hs-CRP, high-sensitivity C-reactive protein

${ }^{*}$ Data presented as mean $\pm \mathrm{SD}$ and count $(\%)$ 


\begin{tabular}{|c|c|c|c|}
\hline & No-Reflow, $(n=63)$ & Normal Reflow, $(\mathrm{n}=334)$ & P value \\
\hline \multicolumn{4}{|l|}{ Infarct-related artery } \\
\hline LAD & $46(73 \%)$ & $254(76 \%)$ & \\
\hline RCA & $15(23.8 \%)$ & $62(18.6 \%)$ & 0.5 \\
\hline LCx & $2(3.2 \%)$ & $18(5.4 \%)$ & \\
\hline \multicolumn{4}{|l|}{ Initial TIMI flow } \\
\hline $\mathbf{0}$ & $56(88.9 \%)$ & $72(21.6 \%)$ & \\
\hline 1 & $7(11.1 \%)$ & $161(48.2 \%)$ & $<0.001$ \\
\hline 2 & 0 & $101(30.2 \%)$ & \\
\hline \multicolumn{4}{|l|}{ Target lesion location } \\
\hline Proximal & $59(93.7 \%)$ & $119(35.6 \%)$ & \\
\hline Medial & $4(603 \%)$ & $169(50.6 \%)$ & $<0.001$ \\
\hline Distal & 0 & $46(13.8 \%)$ & \\
\hline \multicolumn{4}{|l|}{ Type of occlusion } \\
\hline Subtotal & $1(1.6 \%)$ & $225(6704 \%)$ & $<0.001$ \\
\hline Total & $62(98.4 \%)$ & $109(32.6 \%)$ & 0.004 \\
\hline \multicolumn{4}{|l|}{ Lesion type in subtotal occlusion } \\
\hline Concentric & $32(50.8 \%)$ & $105(31.4 \%)$ & \\
\hline Eccentric & $31(49.2 \%)$ & $229(68.9 \%)$ & $<0.001$ \\
\hline Lesion length, mm & $16(14-18)$ & $8(6-10)$ & \\
\hline \multicolumn{4}{|l|}{ Thrombus burden } \\
\hline Low & 0 & $135(40.4 \%)$ & \\
\hline Moderate & $1(1.6 \%)$ & $185(55.4 \%)$ & $<0.001$ \\
\hline High & $62(98.4 \%)$ & $14(4.2 \%)$ & \\
\hline \multicolumn{4}{|l|}{ Method of reperfusion } \\
\hline Balloon angioplasty & 0 & $88(26.3 \%)$ & \\
\hline Direct stenting & $1(1.6 \%)$ & $138(41.3 \%)$ & \\
\hline Stenting + predilation & 0 & $82(24.6 \%)$ & \\
\hline Thrombosuction & $22(34.9 \%)$ & $12(3.6 \%)$ & $<0.001$ \\
\hline Direct stenting + thrombosuction & $10(15.9 \%)$ & $2(0.6 \%)$ & \\
\hline Stenting + predilation + thrombosuction & $18(28.6 \%)$ & $9(2.7 \%)$ & \\
\hline Angioplasty + thrombosuction & $12(19 \%)$ & $3(0.9 \%)$ & \\
\hline GP IIb/IIIa inhibitor & $45(71.4 \%)$ & $113(33.8 \%)$ & $<0.001$ \\
\hline
\end{tabular}

Abbreviations: LAD, left anterior descending; RCA, right coronary artery; LCx, left circumflex artery; GP IIb/IIIa, glycoprotein IIb/IIIa

${ }^{*}$ Data presented as mean (SD) and count (\%)

\begin{tabular}{|c|c|c|c|}
\hline & Coefficient $(\beta)$ & P value & Odds ratio (CI 95\%) \\
\hline Thrombus burden & -5.751 & 0.003 & $0.003(0.001-0.135)$ \\
\hline Lesion length & -0.900 & 0.021 & $0.407(0.189-0.875)$ \\
\hline Time to reperfusion & -4.062 & 0.046 & $0.017(0.001-0.931)$ \\
\hline Type of occlusion & -6.294 & 0.029 & $0.002(0.001-0.519)$ \\
\hline
\end{tabular}

thrombus burden, lesion length, time to reperfusion, and type of occlusion were independently associated with the primary endpoint of the study (Table 4).

\section{Discussion}

The incidence of no-reflow phenomenon has been reported to be $5-24 \%$ in different studies (5-10). In the present study, the rate of no-reflow phenomenon following the primary PCI was $15.9 \%$, which was similar to other studies. This rate was $24.8 \%$ in the study by Kirma et al. (9) and $9.5 \%$ in the one by Du et al. (11).

The results of univariate analysis showed that certain factors, such as age, WBC count, hs-CRP level, time to reperfusion, door to balloon time, type of occlusion (total, subtotal), type of lesion in subtotal occlusions (eccentric, concentric), lesion length, lesion location (proximal, medial, and distal), thrombus burden, and angioplasty technique, could be related to development of no-reflow phenomenon. However, time to reperfusion, type of occlusion, lesion length, and thrombus burden were identified as the independent predictors of no-reflow phenomenon in multivariate analysis.

Generally, prompt restoration of antegrade blood flow of the coronary artery is critical in treatment of Acute MI (AMI). Therefore, primary PCI is superior to thrombolytic therapy (1). One of the most important factors related to no-reflow phenomenon is the reperfusion time (6-10). In the current study, $96.8 \%$ of the patients with no-reflow phenomenon had a reperfusion time more than 4 hours. Previous studies showed that the patients with delayed 
reperfusion time (more than 4 hours) had a 1.4 fold increase in the rate of no-reflow phenomenon (6-10). These patients also had a greater thrombus burden because delayed reperfusion causes the thrombus to be more organized and these organized intracoronary thrombi are very vulnerable to distal embolization during primary PCI. In the early stages of AMI, the main structural component of thrombus is thrombocyte that lyses more easily. With a delayed time to reperfusion, erythrocytes are entrapped within the thrombus and make it firmer. Fragmentation of these thrombi following balloon dilatation can lead to distal embolization. Furthermore, prolonged ischemia can lead to alteration in capillary integrity, edema in capillary bed and myocardial cells, and polymorphonuclear cell plugging $(2$, $3,8-10)$. Although the thrombus burden is a very important issue in the occurrence of no-reflow phenomenon, this phenomenon can occur even in patients with lower burden of thrombus if they have a prolonged reperfusion time. Prolonged ischemia can destroy the capillary bed and it is known that the disruption of the vascular bed is one of the key elements in the pathogenesis of the no-reflow phenomenon $(2,3,6,8-11)$.

Lesion characteristics have also been considered to be an important issue in the no-reflow phenomenon (12). The findings of the present study revealed that type of occlusion, lesion length, and the thrombus burden were independently related to the no-reflow phenomenon. Total occlusion may be a sign of prolonged ischemia and a fully developed thrombus, and causes lower TIMI flow before primary PCI. The TIMI flow before the procedure is one of the key factors implicated in the no-reflow phenomenon (6-11). In our study, all the patients with the no-reflow phenomenon had a TIMI flow $<1$ before the procedure and $89 \%$ showed no antegrade flow (TIMI flow $=0$ ). De Luca et al. (8) and Kirma et al. (9) showed in their studies that the procedural success for primary PCI was better and catheterization complications were lower in the presence of higher TIMI flows prior to PCI. In general, good TIMI flow prior to PCI is a sign of good patency of the infarct-related artery and suggests spontaneous lysis of the thrombus and smaller thrombus burden.

Lesion length could show the plaque burden in an atherosclerotic artery. Kirma et al. (9) reported that the noreflow phenomenon was 5.4 folds higher in the patients with lesion lengths above 13.5 millimeters. In our study, $84.1 \%$ of the patients with no-reflow phenomenon had lesion lengths above 13.5 millimeters, which was significantly higher compared to those without the no-reflow phenomenon.

Thrombus burden is one of the most important factors related to the no-reflow phenomenon. By Intra-Vessel Ultrasound (IVUS) studies, Tanaka et al. (12) and Watanabe et al. (13) demonstrated a marked relationship between the lipid rich plaques and the occurrence of the no-reflow phenomenon. In our study, $98.4 \%$ of the patients with the no-reflow phenomenon had a high thrombus burden, which was more prevalent among the patients with prolonged reperfusion time. According to Ndrepepa G et al. (10) and Zhao et al. (14), thrombus burden is the most important predictor of the no-reflow phenomenon. As discussed earlier, lesions with higher thrombus burdens are vulnerable to distal embolization following balloon predilation.

Generally, AMI can be more complicated and primary PCI may be less successful in the elderly mainly because of their numerous comorbidities and delay in hospitalization (15). In the present study, the patients who had the noreflow phenomenon were almost 10 years older than those without this phenomenon. Advance age was also an independent predictor of the no-reflow phenomenon in the research by Kirma et al. (9). They stated that there was an increased tendency for distal microembolization during primary PCI in the elderly because of their more severe and diffuse coronary atherosclerosis and calcification. However, we found no independent relationships between age and occurrence of the no-reflow phenomenon.

Although the role of inflammatory factors in the noreflow phenomenon has been investigated in many studies, there is no consensus in this regard. In the study by Du et al. (11), both WBC count and hs-CRP were independent predictors of the no-reflow phenomenon. However, the results of multivariate analysis in our study showed no relationships between WBC count and hs-CRP, and noreflow phenomenon.

In conclusion, the findings of the present study indicated that the no-reflow phenomenon after primary PCI would be predictable. Accordingly, the patients with delayed reperfusion, higher thrombus burden, and longer and totally occluded target lesions were more likely to develop the no-reflow phenomenon. Thus, preventive measures, such as using distal protective devices or administration of platelet glycoprotein IIb/IIIa antagonists, are advised to be used in high-risk patients $(16,17)$. Overall, the study results indicated the necessity to perform primary PCI as soon as possible and to reduce the reperfusion time as the most important preventive measures that should be considered in administrative programming for AMI patients.

\section{Acknowledgements}

This research project was financially supported by Rajaie Cardiovascular Medical and Research Center. The authors would like to thank Nasim Naderi and Hooman Bakhshandeh for their scientific support.

\section{Authors' Contribution}

Ata Firouzi was the project supervisor and participated in developing the research idea, study design, data collection, and writing the manuscript. Kamran Aeinfar contributed to study design, data collection, and writing the manuscript. Hamidreza Sanati, Reza Kiani, and Hossein Shahsavari cooperated in data collection and $\mathrm{p}$ reparation of the manuscript.

\section{Financial disclosure}

This article was prepared and published under the technical supervision of ATRIA knowledge-based Company.

\section{Funding/Support}

The study was approved as a research project in Rajaie Cardiovascular Medical and Research Center and the costs for laboratory tests and data collection were provided by this center. 


\section{References}

1. O'Gara PT, Kushner FG, Ascheim DD, Casey DE, Jr., Chung MK, de Lemos JA, et al. 2013 ACCF/AHA guideline for the management of ST-elevation myocardial infarction: executive summary: a report of the American College of Cardiology Foundation/American Heart Association Task Force on Practice Guidelines: developed in collaboration with the American College of Emergency Physicians and Society for Cardiovascular Angiography and Interventions. Catheter Cardiovasc Interv. 2013;82(1):E1-27.

2. Reffelmann T, Kloner RA. The "no-reflow" phenomenon: basic science and clinical correlates. Heart. 2002;87(2):162-8.

3. Reffelmann T, Kloner RA. The no-reflow phenomenon: A basic mechanism of myocardial ischemia and reperfusion. Basic Res Cardiol. 2006;101(5):359-72.

4. Gibson CM, de Lemos JA, Murphy SA, Marble SJ, McCabe CH, Cannon CP, et al. Combination therapy with abciximab reduces angiographically evident thrombus in acute myocardial infarction: a TIMI 14 substudy. Circulation. 2001;103(21):2550-4.

5. Brosh D, Assali AR, Mager A, Porter A, Hasdai D, Teplitsky I, et al. Effect of no-reflow during primary percutaneous coronary intervention for acute myocardial infarction on six-month mortality. Am J Cardiol. 2007;99(4):442-5.

6. Hong YJ, Jeong MH, Choi YH, Ko JS, Lee MG, Kang WY, et al. Predictors of no-reflow after percutaneous coronary intervention for culprit lesion with plaque rupture in infarct-related artery in patients with acute myocardial infarction. J Cardiol. 2009;54(1):36-44.

7. Resnic FS, Wainstein M, Lee MK, Behrendt D, Wainstein RV, Ohno-Machado L, et al. No-reflow is an independent predictor of death and myocardial infarction after percutaneous coronary intervention. Am Heart J. 2003;145(1):42-6.

8. De Luca G, van 't Hof AW, de Boer MJ, Ottervanger JP, Hoorntje JC, Gosselink AT, et al. Time-to-treatment significantly affects the extent of ST-segment resolution and myocardial blush in patients with acute myocardial infarction treated by primary angioplasty. Eur Heart J. 2004;25(12):1009-13.

9. Kirma C, Izgi A, Dundar C, Tanalp AC, Oduncu V, Aung SM, et al. Clinical and procedural predictors of no-reflow phenomenon after primary percutaneous coronary interventions: experience at a single center. Circ J. 2008;72(5):716-21.

10. Ndrepepa G, Tiroch K, Keta D, Fusaro M, Seyfarth M, Pache J, et al. Predictive factors and impact of no reflow after primary percutaneous coronary intervention in patients with acute myocardial infarction. Circulation: Cardiovascular Interventions. 2010;3(1):27-33.

11. Du J, Chen S, Wang W, Zhou C, Li G, Huang T, et al. hs-CRP is a potential predictor of no-reflow in patients with AMI after emergency PCI. Journal of Geriatric Cardiology December. 2008;5(4):217.

12. Tanaka A, Kawarabayashi T, Nishibori Y, Sano T, Nishida Y, Fukuda $\mathrm{D}$, et al. No-reflow phenomenon and lesion morphology in patients with acute myocardial infarction. Circulation. 2002;105(18):2148-52.

13. Watanabe T, Nanto S, Uematsu M, Ohara T, Morozumi T, Kotani $\mathrm{J}$, et al. Prediction of no-reflow phenomenon after successful percutaneous coronary intervention in patients with acute myocardial infarction: intravascular ultrasound findings. Circ J. 2003;67(8):667-71.

14. Zhao Y, Chen Y, Tian F, Wang C, Hu S, Wang J, et al. [Predictors of the no-reflow phenomenon after primary percutaneous coronary intervention for acute myocardial infarction]. Nan Fang Yi Ke Da Xue Xue Bao. 2012;32(2):261-4.

15. Singh M, Mathew V, Garratt KN, Berger PB, Grill DE, Bell MR, et al. Effect of age on the outcome of angioplasty for acute myocardial infarction among patients treated at the Mayo Clinic. Am J Med. 2000;108(3):187-92.

16. Niccoli G, Kharbanda RK, Crea F, Banning AP. No-reflow: again prevention is better than treatment. Eur Heart J. 2010;31(20):2449-55.

17. Niccoli G, Marino M, Spaziani C, Crea F. Prevention and treatment of no-reflow. Acute Card Care. 2010;12(3):81-91. 